\title{
“O Equilibrista”: Atuação do Psicólogo no NASF no Vale do Itajaí
}

\author{
Roberta Borghetti Alves ${ }^{1}$ \\ ${ }^{1}$ Universidade do Vale do Itajaí, SC, Brasil. \\ Natália de Oliveira Bruning ${ }^{1}$ \\ ${ }^{1}$ Universidade do Vale do Itajaí, SC, Brasil. \\ Ketillyn Cristina Kohler ${ }^{1}$ \\ ${ }^{1}$ Universidade do Vale do Itajaí, SC, Brasil.
}

Resumo: Dentre as possibilidades de atuação na rede pública de saúde, o Núcleo de Apoio a Saúde da Família (NASF) surge como um espaço para o psicólogo atuar na Atenção Primária. Desse modo, esta pesquisa teve como objetivo compreender a atuação do psicólogo inserido no NASF na Região de Saúde da Foz do Vale do Itajaí, em Santa Catarina. Foi aplicado um roteiro de entrevista semiestruturada com psicólogos atuantes no NASF na referida região e seus respectivos dados foram analisados por meio da Grounded Theory, com o auxílio do software Atlas Ti na versão 7.0. Destaca-se como principais categorias de análise o território, o apoio e o cuidado. Através desses resultados, identificou-se que a atuação do psicólogo neste serviço está voltada para as práticas sanitária, técnico-pedagógica e clínico-assistencial. Os resultados apontaram uma grande demanda de saúde mental, assim, sugere-se a criação de polos multidisciplinares para dar a retaguarda aos profissionais do NASF.

Palavras-chave: Atenção Primária à Saúde, Psicologia, Políticas Públicas, Núcleo de Apoio à Saúde da Família, Saúde Mental.

\section{“The Equilibrist": Performance of the Psychologist in the NASF in the Vale of Itajaí}

\begin{abstract}
Among the possibilities of acting in the public health network, the Family Health Support Center (NASF) appears as a space for the psychologist, to act in Primary Care. Thus, this research aimed to understand the performance of the psychologist inserted in the NASF in the Health Region of Foz do Vale do Itajaí, in Santa Catarina. A semi-structured interview script was applied with NASF psychologists in that region and their respective data were analyzed through Grounded Theory with the assistance of Atlas Ti software in version 7.0. The main categories of analysis are the territory, the support and the care. Through these results, it was identified that the psychologist's performance in this service is focused on sanitary, technical-pedagogical and clinical-assistance practices. The results pointed out a great demand for mental health, thus, this document suggests to create multidisciplinary poles to give some support to professionals of NASF.
\end{abstract}

Keywords: Primary Health Care, Psychology, Public Policies, Family Health Support Center, Mental health. 


\title{
“El Equilibrista”: Actuación del Psicólogo en el NASF en el Valle do Itajaí
}

\begin{abstract}
Resumen: Entre las posibilidades de actuación en la red pública de salud, el Núcleo de Apoyo a la Salud de la Familia (NASF) surge como un espacio para que el psicólogo actúe en la Atención Primaria. De este modo, esta investigación tuvo como objetivo comprender la actuación del psicólogo insertado en el NASF en la Región de Salud de Foz do Vale do Itajaí, en Santa Catarina. Se aplicó un guion de entrevista semiestructurada con psicólogos actuantes en el NASF en dicha región y sus respectivos datos fueron analizados a través de la Grounded Theory, con la ayuda del software Atlas Ti en la versión 7.0. Se destaca como principales categorías de análisis el territorio, el apoyo y el cuidado. A través de estos resultados, se identificó que la actuación del psicólogo en este servicio está orientada a las prácticas sanitarias, técnico-pedagógica y clínico-asistencial. Los resultados apuntaron una gran demanda de salud mental, así, se sugiere la creación de polos multidisciplinarios para dar la retaguardia a los profesionales de NASF.
\end{abstract}

Palabras clave: Atención Primaria a la Salud, Psicología, Políticas públicas, Núcleo de Apoyo a la Salud de la Familia, Salud mental.

\section{Introdução}

A inserção do psicólogo, inicialmente, era predominante nos contextos clínicos, escolares e organizacionais, de modo que só posteriormente a Psicologia obteve espaço na saúde pública. Dentre as possibilidades de atuação na rede pública de saúde, o Núcleo de Apoio à Saúde da Família (NASF) surge como um espaço de inserção para o psicólogo e outros profissionais de saúde atuarem na Atenção Primária (Furtado, \& Carvalho, 2015).

O NASF foi regulamentado por meio da Portaria $\mathrm{n}^{\mathrm{o}} 154$, de 24 de janeiro de 2008, com o intuito de apoiar e complementar o trabalho realizado pelas equipes de Estratégia Saúde da Família (ESF), para ampliar as ações da Atenção Primária e aumentar a resolutividade no cuidado dos usuários (Portaria GM n. 154, 2008). Formado por uma equipe de diferentes profissionais de saúde, o NASF prevê que a prática dos processos de referência e contrarreferência dos encaminhamentos, seja substituído pelo processo de atendimento compartilhado e longitudinal, a fim de corresponsabilizar todos os profissionais envolvidos no caso (Brasil, 2014).

A partir da lógica do apoio matricial, a equipe de referência (ESF) é responsável pela condução dos casos, e os profissionais apoiadores (NASF) tem como função construir um espaço de comunicação e compartilhamento de conhecimento entre ambas equipes para aumentar a capacidade resolutiva dos problemas de saúde do território (Campos, \&
Domitti, 2007). Tendo em vista a crescente demanda de saúde mental, o Ministério da Saúde preconiza que pelo menos um profissional de saúde mental esteja inserido no NASF, de modo a possibilitar a inserção do psicólogo na Atenção Primária (Portaria GM n. 154, 2008).

A atuação do psicólogo no NASF está sustentada em três propostas articuladas, sendo estas: intervenções sanitárias, clínico-assistencial e técnico-pedagógica. A intervenção sanitária refere-se ao levantamento das demandas do território, onde a partir da avaliação das demandas será possível estabelecer prioridades e viabilizar projetos de intervenção que atendam à necessidade dos usuários. A dimensão técnico-pedagógica tem como intuito empoderar as ESF para realizar o acolhimento dos usuários que estão em risco psicossocial, promovendo um espaço de diálogo e discussão conjunta dos casos clínicos, a fim de negociar entre as equipes de referências as demandas prioritárias, bem como realizar ações de educação permanente para efetivar uma pauta de trabalho. Já a proposta clínico-assistencial engloba atendimentos individuais, compartilhados e grupais, mas a atuação clínica deve ser somente uma das intervenções realizadas pelos psicólogos, sem fazer com que toda a demanda se torne apenas objeto clínico. Para que isso aconteça, é necessário fazer uma negociação e uma pactuação com as ESF para se estabelecer uma relação de cuidado em saúde (Lucena, Mourão, \& Nepomuceno, 2009). 
Para verificar o estado da arte da temática foi realizada uma revisão bibliográfica no portal BVS-Psi, na base de dados PePSIC e SciELO utilizando os descritores: "atuação Psicologia"; "Núcleo de Apoio à Saúde da Família"; "atuação psicólogo + NASF". A definição dos critérios para seleção de artigos da pesquisa foi definida através do parâmetro linguístico português e tratar-se de uma pesquisa empírica, com destaque na atuação do psicólogo no NASF. Foram selecionados sete artigos científicos e através desses resultados foi possível observar que há o predomínio de pesquisas com a abordagem qualitativa, e que o roteiro de entrevista semiestruturada é o principal instrumento de coleta de dados.

Acerca dos principais resultados, destacam-se como potencialidades da atuação do psicólogo os profissionais que realizam uma atuação abrangente, que visa suprir a demanda do território, ações efetivas de apoio matricial e ampliação dos aspectos que envolvem o cuidado à saúde do usuário. As fragilidades englobam a formação acadêmica com ênfase na atuação da clínica tradicional, o alto número de ESF pelas quais o NASF é responsável, relações ainda verticalizadas com a ESF e com a gestão, e dificuldade de articulação entre ESF e NASF.

Dentre os artigos científicos disponíveis que abordam sobre o tema, nenhum deles discorre sobre a atuação destes profissionais na Região de Saúde da Foz do Vale Itajaí, fazendo deste trabalho colaborador para a área científica estudada. A pesquisa também poderá contribuir para reflexão da atuação do psicólogo nesse núcleo, de modo a trazer a realidade dos serviços existentes na Região de Saúde da Foz do Vale do Itajaí, e a partir disso destacar os aspectos que são concretizados na prática e aqueles que precisam ser revisados, a fim de que o apoio matricial possa ser potencializado.

Com base nestas reflexões, este estudo teve como objetivo geral compreender a atuação dos psicólogos inseridos no NASF da Região de Saúde da Foz do Vale do Itajaí. E a partir disso, buscou-se explorar as ações sanitárias, descrever as atividades técnico-pedagógicas e identificar as intervenções clínico-assistenciais praticadas pelos psicólogos inseridos no NASF.

\section{Método}

\section{Delineamento da pesquisa}

Este estudo trata-se de uma pesquisa descritiva-exploratória de natureza aplicada, pois tem o intuito de descrever e explorar o processo de trabalho dos psicólogos inseridos no NASF, proporcionando o levantamento de opiniões, atitudes e crenças dos profissionais dessa área diante o tema estabelecido. Possui abordagem qualitativa, que foi realizada por meio de pesquisa de campo, para aprofundar o tema abordado, de forma que siga os objetivos estabelecidos (Pope, \& Mays, 2009).

\section{Participantes}

A proposta da pesquisa era de entrevistar todos psicólogos que atuavam no NASF na Região de Saúde da Foz do Vale do Itajaí, no entanto não foi possível realizar a coleta de dados com um participante, pois uma Secretaria Municipal de Saúde não retornou acerca da possibilidade de tal coleta. Desse modo, foram entrevistados nove psicólogos que atuam no NASF da referida região.

Dentre os nove participantes, sete do sexo feminino e dois do sexo masculino, com a média de idade de 38 anos e sete meses. Em relação à formação profissional, cinco possuem pós-graduação e/ou mestrado em outra área, dois participantes possuem pós-graduação na área de saúde pública e dois não realizaram especialização. $\mathrm{O}$ ingresso dos psicólogos no NASF ocorreu por meio de concurso público, a média do tempo de inserção no NASF é de um ano e cinco meses, sendo cinco participantes com carga horária de 40 horas semanais, dois com 30 horas e dois com 20 horas semanais.

Em relação à modalidade do NASF, oito deles estão incluídos no NASF 1 e apenas um participante está incluído no NASF 2. No que consiste ao número de equipes de ESF apoiadas pelo NASF, identificou-se que um psicólogo apoia dez equipes, três psicólogos apoiam oito equipes, um psicólogo apoia seis equipes, um apoia quatro equipes. Entretanto, três psicólogos apontaram que o apoio às ESF não acontece, pois o NASF está cadastrado somente no Cadastro Nacional de Estabelecimentos de Saúde (CNES), e seus respectivos profissionais estão alocados na atenção secundária. Em meio a esses dados, também foi possível identificar que na referida região o NASF que apoia dez equipes está com o número de ESF apoiadas superior ao que é regulamentado pela Portaria $\mathrm{n}^{\circ}$ 3.124(2012), tendo em vista que a modalidade de NASF 1 deve estar vinculada com no mínimo cinco e no máximo nove equipes de ESF (Brasil, 2012). 


\section{Instrumento de coleta de dados}

Para realizar a coleta de dados, foi elaborado um roteiro de entrevista semiestruturada, dividido em cinco tópicos norteadores, sendo eles: dados sociodemográficos do profissional, aspectos que envolvem a caracterização do NASF, intervenções clínica-assistencial, técnico-pedagógica e sanitária, que permeiam a atuação do psicólogo no NASF.

\section{Procedimentos de coleta de dados e éticos}

Na primeira etapa foi entrado em contato com a Secretaria Estadual de Saúde, a fim de verificar quantos NASF estavam implantados na Região de Saúde da Foz do Vale do Itajaí e qual sua localidade. A etapa seguinte consistiu no contato com as Secretarias Municipais de Saúde, com o intuito de solicitar a autorização para realizar a coleta de dados. Posteriormente a pesquisa foi submetida ao Comitê de Ética da Universidade do Vale do Itajaí (Univali) e mediante o aceite sobre o protocolo $\mathrm{n}^{\circ}$ 1.951.016 (CAAE 62214916.6.0000.0120) foi realizado o contato com os psicólogos inseridos nos NASF. A participação da pesquisa foi de caráter voluntário, sendo garantido, através do Termo de Consentimento Livre e Esclarecido (TCLE), o anonimato dos participantes, bem como a ausência de remuneração frente a participação da pesquisa.

\section{Análise e discussão dos dados}

Para a análise de dados foi utilizada a Grounded Theory ou Teoria Fundamentada nos Dados, que de acordo com Cassiani e Almeida (1999), é uma teoria que consiste em auxiliar os pesquisadores, esclarecendo aspectos relevantes na fase de análise de dados. Nesta pesquisa foi aplicado às fases de codificação seletiva, no qual, buscou-se classificar os temas importantes trazidos pelos entrevistados transformando-os em categorias, subcategorias e elementos temáticos. Para auxílio da codificação de dados foi utilizado o software Atlas.ti na versão 7.0, que auxiliou explorar os fenômenos mais complexos dos dados, permitindo atingir uma representação de conteúdo mais relevante, de acordo com os objetivos da pesquisa (Friese, 2013).

\section{Resultados e discussões}

Para compreender as práticas sanitária, técnico-pedagógica e clínico-assistencial dos psicólogos que atuam nos NASF na Região de Saúde da Foz do Vale do Itajaí, a análise foi dividida em três categorias: território, apoio e cuidado. Neste artigo foram discutidos os principais resultados encontrados na pesquisa, evidenciados nas falas dos psicólogos entrevistados.

$\mathrm{Na}$ categoria território foram analisados os indicadores de saúde mental, o índice expressivo de pessoas que fazem uso de psicotrópicos e a relação do psicólogo e comunidade. No apoio obteve-se um conjunto de elementos temáticos que englobam a educação permanente, a inter-relação com a ESF, as reuniões de equipe, o Projeto Terapêutico Singular, discussão de casos e ações intersetoriais. Na última categoria, o cuidado compreendeu-se os grupos, os atendimentos individuais e compartilhados e a necessidade de haver retaguarda profissional para a Psicologia.

\section{Categoria 01 - Território}

Para a categoria território foi objetivado explorar as ações sanitárias realizadas pelos psicólogos entrevistados, sendo elas, o reconhecimento do território, a relação do psicólogo com a comunidade, e a forma com que é realizado o levantamento e monitoramento de indicadores e demandas territoriais, no que consiste à saúde mental. De acordo com Santos e Rigotto (2011), o território deve ser pensado além da sua delimitação no espaço, ele deve ser compreendido como algo que está em constante construção e transformação, através da sua estrutura histórica, política, social e cultural, de forma a abarcar sua complexidade. Assim, o território torna-se um importante espaço para articular o protagonismo do cuidado, bem como para realizar o levantamento de interesses e necessidades da população, a fim de realizar os planejamentos das ações a serem realizadas pelas equipes de saúde.

Nesse sentido, a análise da subcategoria reconhecimento das peculiaridades do território foi delimitada, de modo a conter como elemento temático a relação entre o psicólogo e a comunidade, na qual os participantes enfatizaram que conhecer o contexto em que os usuários vivem propicia maior subsídio para os psicólogos atuarem a partir realidade vivenciada pela comunidade. Santos e Rigotto (2010) reforçam essa ideia quando citam que nos Sistemas de Saúde é necessário desvelar as relações com o ambiente, o reconhecimento dos territórios e seus contextos. Isso reforça a fala dos psicólogos entrevis- 
tados, que comentam sobre a importância de conhecer o contexto em que a população vive para planejar as estratégias de trabalho.

Outros aspectos ressaltados na fala dos profissionais são a chegada do psicólogo no território e a descoberta da comunidade por esse novo profissional, quando este é um profissional a que muitos não tiveram acesso anteriormente e agora podem ter, a partir da constituição dos NASF. Nesse sentido, Furtado e Carvalho (2015) salientam que o reconhecimento da comunidade é fundamental para que as equipes de NASF e ESF consigam ampliar o vínculo entre os usuários e os profissionais de saúde, como comentado: "Eu acho que a comunidade tá descobrindo esse profissional que ele nunca teve acesso e está possibilitando bons resultados. Acho que ela tá se sentindo mais próxima" (Psicólogo 05).

A partir da chegada do psicólogo no território, os participantes relataram que realizam o levantamento de indicadores de saúde mental e de demandas do território, através do trabalho realizado em conjunto com as ESF. Destaca-se como estratégias utilizadas: educação permanente com a ESF, na qual relata as principais demandas do território; aplicação de questionário com os profissionais da ESF; diálogo com profissionais inseridos anteriormente no NASF e com os profissionais da ESF; e utilização do prontuário eletrônico como meio de verificar as demandas prevalentes. Identifica-se que as estratégias utilizadas pelos profissionais entrevistados se voltam ao levantamento de indicadores, por meio da percepção da equipe de ESF, sem haver o levantamento diretamente com a população adscrita.

Salvo das estratégias indicadas acima, percebe-se dificuldade na organização e planejamento para levantamento e monitoramento dos indicadores de saúde voltados à saúde mental, já que os dados às vezes não são identificados pelas equipes. Esses aspectos corroboram com os resultados encontrados por Reis, Medeiros, Pacheco, e Caixeta (2016), que afirmam que os profissionais que atuam no NASF percebem o déficit em ferramentas que favoreçam a consolidação dos dados territoriais e, consequentemente, acabam prejudicando o diagnóstico da área adscrita como de responsabilidade das equipes de ESF e NASF, de modo a limitar as propostas voltadas para a realidade dos usuários.

De acordo com os entrevistados, um dos fatores que está favorecendo o levantamento de demandas de saúde mental é o Programa Nacional de Melhoria do Acesso e de Qualidade da Atenção Básica (PMAQ-AB). Desta forma, as equipes de ESF e NASF estão se mobilizando para organizar os indicadores de saúde, dentre eles, o de saúde mental, já que o PMAQ solicita que a Atenção Primária tenha esses dados para organizar seu trabalho a partir das demandas de seu território. De acordo com o manual instrutivo do PMAQ, para realizar a gestão do cuidado, as equipes de Atenção Primária devem organizar seu processo de trabalho a partir do cadastro dos usuários do seu território, identificando os problemas de saúde vivenciados pela comunidade, para assim, propor estratégias que orientem as intervenções realizadas pela equipe (Brasil, 2012).

Em relação aos indicadores de saúde mental, quatro entrevistados destacaram as demandas voltadas a ansiedade, a depressão e as queixas escolares. Em relação à queixa escolar, os autores Cord, Gesser, Nunes e Storti (2015) afirmam que ainda as escolas continuam solicitando intervenções dos profissionais de saúde voltadas ao diagnóstico e medicalização das crianças, de modo a culpabilizar os alunos pelas dificuldades de aprendizagem. Tavares (2008) aponta que o profissional de saúde mental, quando vinculado à Atenção Primária por meio do NASF, poderia promover a realização de diagnósticos interventivos dos riscos psicossociais vivenciados pelos usuários de maneira precoce, podendo este ser um eixo norteador para o psicólogo realizar suas ações na comunidade, porém as autoras dessa pesquisa questionam a real concretização da sugestão de Tavares, pois identificou-se uma sobrecarga de trabalho dos psicólogos do NASF que atuam na Região de Foz do Vale do Itajaí e necessidade de articulação com as políticas públicas.

A partir dos indicadores de saúde mental, os profissionais identificaram um índice expressivo de usuários que fazem uso de psicotrópicos, no qual se demonstram preocupados com uso demasiado desse tipo de medicação e com a forma com que são realizados os atendimentos pela equipe de referência aos usuários em sofrimento psíquico. Tais ações caracterizam-se pela troca de receitas e de encaminhamentos para outros serviços. Esse resultado vai ao encontro da pesquisa de Gryschek e Pinto (2015), em que identificaram que as equipes de ESF têm dificuldades de atender as demandas de saúde mental, assim, geralmente realizam a prescrição de medicação e o encaminhamento para serviços especializados. 


\section{Categoria 02 - Apoio}

Para a categoria apoio, foram destacadas as ações em conjunto com as equipes que dão subsídio às mesmas, considerando o apoio técnico-pedagógico que define como ações relacionadas à troca de experiências das equipes, discussão de casos e necessidades em comum (Gonçalves, Lancman, Sznelwar, Cordone, \& Barros, 2015). Os entrevistados destacaram ações realizadas nas questões de apoio técnico-pedagógico, como educação permanente, a inter-relação com a ESF, as reuniões de equipe, o Projeto Terapêutico Singular, discussão de casos e ações intersetoriais.

$\mathrm{Na}$ subcategoria de educação permanente, os profissionais destacam a atuação com os profissionais da ESF voltadas para a mudança do processo de trabalho da equipe, no qual citam a realização de dinâmicas de equipe, a discussão sobre os procedimentos e as estratégias a serem utilizados com os usuários, sendo prioritariamente realizadas nas reuniões entre NASF e ESF. Constatou-se que, embora haja dificuldade em acolher e atender os usuários com o sofrimento psíquico, a educação permanente tem sido uma importante ferramenta para a gestão do cuidado de tais usuários, de modo a possibilitar maior segurança para a equipe acolher esta demanda. Segundo Cezar, Rodrigues e Arpini (2015), a educação permanente é uma proposta que deve se tornar na rede pública uma ação capaz de superar as práticas desarticuladas das equipes de ESF, além de mediar a articulação entre profissionais da rede de saúde. Essas práticas são destacadas pelos profissionais, conforme exemplifica-se abaixo:

As equipes têm receio de orientar ou de acolher o paciente, e às vezes tu dando uma orientação para equipe "é desse jeito, é assim" daí a equipe se sente confiante de ficar atendendo aquele paciente e não necessariamente tem que encaminhar para psiquiatria ou para o atendimento clínico (Psicólogo 08).

Além da análise sobre educação permanente, os profissionais ressaltaram a inter-relação com as equipes de ESF, na qual foram apontadas, pelos entrevistados, as potencialidades e as fragilidades. Ao atuar juntamente com a ESF, destacam-se como potencialidades a experiência e o conhecimento que as ESF têm em relação aos usuários, ressaltando-se, principalmente, a atuação do agente comunitário de saúde. Sua atuação proporciona uma ampliação do olhar para o profissional de Psicologia ao adentrar no território de sua abrangência, além de possibilitar um espaço de aprendizagem, de troca de visões e de concepções que o trabalho em conjunto proporciona. Da mesma forma, a pesquisa de Andrade et al. (2012) destaca a possibilidade do trabalho em equipe como uma potencialidade das ações realizadas em conjunto entre ESF e o NASF.

Como fragilidades, os entrevistados citam que a ESF ainda tem dificuldade no entendimento sobre o que é NASF. Além disso, para as equipes dos demais pontos de atenção, o NASF é visto como um serviço voltado a "apagar incêndio", pois eles são solicitados apenas para encaminhar usuários a alguma outra especialidade. Essas dificuldades geram, como mencionado, fragilidades na comunicação, inexistência de diálogo, dificuldade em trabalhar em equipe, como citado na fala do psicólogo 04: "As fragilidades realmente é a comunicação, [...], quando eles não sabem o que fazer chamam o NASF, ou às vezes chama o NASF para encaminhar para algum setor, depende da unidade ainda não se tem o entendimento do NASF".

A fala do psicólogo acima corrobora com a pesquisa de Andrade et al. (2012), que apontou, como fragilidade encontrada, a necessidade de entender o processo de trabalho de ESF e NASF, já que, segundo alguns profissionais, o NASF ainda é entendido como um serviço de referência, e não como de suporte à ESF, e isso propicia a sobrecarga dos profissionais.

Além dos desafios citados, foram identificados outros pontos relevantes em suas falas, como problemas de equipes consolidadas, rotatividade de profissionais, o fato de o agente comunitário de saúde ser vizinho da população que realiza o atendimento domiciliar, de modo a sobrepor sua percepção pessoal ao invés da profissional, o que dificulta o relacionamento entre equipe e agente comunitário de saúde. Destacam-se ainda, a dificuldade de levantar demandas com a equipe e o desconhecimento das equipes de ESF sobre a sua própria atuação.

Três profissionais citam também que o apoio às equipes é inexistente, porque o NASF só existe no Cadastro Nacional de Estabelecimento de Saúde (CNES). Esses resultados favorecem com o que foi identificado pelas autoras Cezar et al. (2015), quando citam os desafios dos profissionais de Psicologia dentro do Sistema Único de Saúde (SUS), existindo a dificuldade de outros profissionais verem a Psicologia 
como atuante nesses espaços, acreditando que os psicólogos atuam apenas em saúde mental e que essa atuação é exclusiva deste profissional.

Outro elemento levantado nas falas dos entrevistados foram as reuniões em equipe, que representam outra forma de ação em conjunto com os profissionais, na qual eles podem sugerir a pauta das reuniões e realizar o planejamento das intervenções. Citam também que conseguem levar temas para serem discutidos em conjunto, mas que o espaço da reunião é vago, pois o tempo é limitado. Para que o NASF se apresente resolutivo, é necessário estabelecer espaços para reunião de equipe para planejar ações, discutir casos, definir critérios de encaminhamento ou compartilhamento de casos, resolução de conflitos, entre outras atividades (Andrade et al., 2012). Além disso, torna-se necessário garantir um espaço para que os profissionais do NASF possam de fato participar das reuniões.

Além dos supracitados, os entrevistados destacaram algumas das ações intersetoriais que realizam no NASF. Dentre as ações realizadas, um participante apontou que realiza trabalho em conjunto com a equipe escolar, juntamente com a equipe multiprofissional do NASF, com o intuito de diminuir a demanda dos atendimentos clínicos, conforme é apresentado no relato abaixo:

Junto com a escola, a gente planejou um grupo de escuta, com a fono, a psico e a nutricionista. A princípio, fomos nós 3 , junto com as orientadoras da escola e a professora de apoio, então todo mês a gente se reúne para conversar sobre situações que inicialmente elas iriam encaminhar para o serviço especializado (Psicólogo 03).

A ação realizada na escola vai ao encontro da intersetorialidade, que busca considerar as condições de vida do usuário para desenvolver intervenções que integrem outros setores, garantindo a integralidade na atenção ao usuário (Brasil, 2014). Outras atividades como inserção em outros projetos também são realizadas nas escolas, conforme preconiza o Programa Saúde na Escola (PSE), porém os psicólogos citam que ainda existe dificuldade de abertura da escola para algumas intervenções das equipes ESF e NASF.

Considerando as ações de apoio para os profissionais da ESF, os psicólogos dialogam sobre a importância em participar da construção do Projeto
Terapêutico Singular (PTS) e das discussões de caso. Foi possível compreender que os profissionais dão importância às discussões de casos entre as equipes de ESF e NASF, pois essa estratégia possibilita que o trabalho ocorra de maneira efetiva, propiciando a ampliação, compartilhamento e construção conhecimento. Esses dados corroboram com os resultados na pesquisa realizada por Azevedo e Kind (2013), em que os entrevistados destacaram as discussões de casos como um momento importante para a troca de saberes e construção de vínculo entre as equipes de ESF e NASF.

No entanto, identifica-se nas falas dos entrevistados dificuldades na construção do Projeto Terapêutico Singular, tendo em vista que algumas ESF não querem utilizar essa ferramenta para que a Unidade Básica de Saúde (UBS) não se torne um local de referência para o usuário. Perrella (2015), em sua pesquisa afirma que os profissionais de saúde ainda têm dificuldades de inserir o PTS nas discussões entre as equipes de ESF e NASF, mesmo reconhecendo a importância desta ferramenta. Percebeu-se também que alguns profissionais das outras áreas possuem resistência para debater casos e experiências, dificultando a inclusão desta ferramenta no processo de trabalho.

\section{Categoria 03 - Cuidado}

A categoria cuidado foi construída com base nas intervenções grupais, atendimentos individuais, atendimentos compartilhados e na necessidade de haver retaguarda profissional, a partir da perspectiva clínico-assistencial. Foram identificadas atividades voltadas para educação em saúde, nas quais os profissionais realizam ações de prevenção de doenças e promoção de saúde nos territórios, campanhas, ações na sala de espera de atendimentos e em diversas modalidades de grupos. Entende-se como promoção de saúde um conjunto de estratégias e modos de produção de saúde que tem como intuito atender as demandas sociais de saúde, em esferas individuais e coletivas, de modo que seja garantido a melhoria da qualidade de vida dos usuários (Malta et al., 2016).

Em relação às intervenções grupais, os entrevistados apontaram que realizam grupos de convivência, grupos voltados para mulheres no intuito de possibilitar suporte e apoio, grupos operativos que buscam atingir uma tarefa que possuem em comum, tais como redução ou parada do uso do tabaco e controle da hipertensão e da diabetes, contribuindo na melho- 
ria da qualidade de vida. Os profissionais salientam a importância da atuação multiprofissional nos grupos, seja com os profissionais do NASF ou com a equipe da ESF. A atuação multiprofissional é exemplificada no relato do participante abaixo:

A gente tem grupo de idosos [...] e acaba sendo multiprofissional, vai lá a fisio falar de movimento do corpo, prevenção há quedas, vai a nutricionista alimentação saudável, vai educação física fazer uma atividade com elas e a Psicologia [...], que acaba influenciando na saúde física e biológica e do corpo das pessoas né (Psicólogo 04).

Identifica-se no relato supracitado que há um trabalho multidisciplinar, no qual cada profissional aborda uma temática que é de sua especialidade. As autoras Azevedo e Kind (2013), em sua pesquisa, identificaram que os psicólogos percebem que sua atuação deve ultrapassar a multidisciplinaridade, intervindo de maneira interdisciplinar, tendo em vista que a interdisciplinaridade é uma vertente essencial para atuação no NASF, de modo a propiciar o trabalho com diferentes profissionais nas intervenções grupais.

Quanto aos grupos psicoterapêuticos, oito profissionais citam que não realizam essa atividade, porém comentam a possibilidade de existir grupos voltados para crianças, egressos do Centro de Atenção Psicossocial (CAPS), gestantes, adolescentes e com pais de crianças com sofrimento psíquico. Apesar da existência de grupos, a resistência dos usuários em participar de grupos é enfatizada, onde existe a baixa adesão ou o esvaziamento a longo prazo.

A baixa adesão nos grupostambém foi um resultado encontrado na pesquisa de Hori e Nascimento (2014), no qual as equipes de saúde mental dos NASF encerraram grupos devido à falta de adesão. De acordo com os participantes, os motivos relacionados à baixa participação em grupos envolvem questões culturais da região, dificuldade em se expor, o requerimento somente para atendimento clínico, ou ainda porque o tema não vai ao encontro do interesse dos usuários. Desse modo, salienta-se a necessidade de repensar o grupo psicoterapêutico, a fim de que ele seja potencializado.

Ainda com relação aos grupos, verificou-se nas falas dos participantes que a formação em Psicologia ainda possui defasagem voltada a condução e realização de grupos. Esses resultados estão em convergên- cia com o que foi encontrado na pesquisa de Azevedo e Kind (2013), na qual os psicólogos apontam que durante sua formação não tiveram preparação suficiente para realizar trabalho com grupos. Isso reflete na dificuldade que os profissionais possuem na condução de grupos. Em relação às vantagens, citam que a realização de grupos diminuiria as demandas para atendimentos individual, mas pelo fato de as pessoas não aderirem os grupos, se torna um desafio, pois mobiliza o profissional a mostrar o seu trabalho e propor outros caminhos além do atendimento individual.

Outra subcategoria analisada, a partir das entrevistas com os profissionais, foram os atendimentos individuais e domiciliares. Sete psicólogos citaram que realizam os atendimentos individuais e um deles cita que a comunidade pede por atendimento individual, e não adere a grupos ofertados, como já salientado. Além disso, os psicólogos enfrentam a alta demanda de crianças e adolescentes encaminhados pelas escolas, encaminhamentos sem muitas informações, discordância com a equipe sobre clínica individual e dificuldade de romper a cultura de atendimento clínico.

Em relação aos processos de atendimento individual, foi identificado que quatro dos profissionais realizam atendimentos mais pontuais quando a ESF solicita apoio, como forma de realizar uma triagem para encaminhar o usuário para outro serviço, ou atendem algumas demandas através da psicoterapia breve. Os profissionais também mencionaram que possuem uma grande lista de espera para atendimento clínico, já que os usuários preferem por essa forma de atendimento. Com relação a sua atuação, foi referido que os psicólogos sentem-se, muitas vezes, "apagando o fogo", como mencionado:

A estratégia de saúde da família é muito rápida, até tenho vontade de explorar mais os casos, mas não tenho tempo, você tem que apagar mesmo o fogo daquele momento, e quando resolve aquele problema, geralmente a própria pessoa acaba não vindo mais. Não é como a clínica clássica (Psicólogo 07).

O caderno de Atenção Básica do NASF cita que o atendimento individual específico, diante a lógica de apoio matricial, não se exclui como prática realizada pelo psicólogo do NASF. No entanto, as ações do profissional nesses espaços devem estar voltadas também para compartilhar o cuidado, de forma que 
se discuta com outros profissionais os diversos tipos de possibilidades de atuação, além da clínica, a fim de diminuir a sobrecarga de trabalho (Brasil, 2014).

No que diz respeito aos atendimentos domiciliares, verificou-se que cinco dos profissionais pesquisados realizam essa modalidade de atendimento quando a equipe da ESF solicita apoio, e que nesses atendimentos é priorizado que vá algum profissional da ESF e ACS da área, juntamente com o profissional do NASF. Azevedo e Kind (2013) identificaram em sua pesquisa que a solicitação por atendimentos domiciliares ocorre com grande frequência.

Já nessa pesquisa, três profissionais relataram que não realizam atendimento domiciliar, e uma participante destacou que não realiza essa modalidade de atendimento porque não é disponibilizado o carro da Secretaria Municipal de Saúde. Lancman, Gonçalves, Cordone e Barros (2013), apresentaram em sua pesquisa que os profissionais de saúde tinham que utilizar o seu próprio meio de locomoção para realizar as intervenções. Verificou-se também que os psicólogos realizam atendimentos compartilhados, de acordo com a solicitação das equipes da ESF, conforme consta na fala do psicólogo 03: "Hoje a gente tem uma parceria bem legal com a equipe da estratégia consultas compartilhadas, não só com a médica, mas também com a enfermeira também, com os outros profissionais do NASF".

A consulta compartilhada é uma ferramenta de trabalho indicada pelo Caderno de Atenção Básica NASF, sendo destacada como uma oportunidade de ter um contato entre usuário e equipe de apoio, necessitando de pelo menos dois profissionais atendendo juntos, além do usuário ou seus familiares (BRASIL, 2014). Essa atividade foi citada por quatro profissionais, porém comentam ainda sobre os desafios no trabalho com profissionais de outras áreas, como a fala referida: "Porque fiz com a médica, a atuação dela é diferente da minha. Ela era muito diretiva, um pouco agressiva até na atuação dela, mas fizemos, ela atendeu o meu pedido, no final" (Psicólogo 08).

Outro aspecto importante que emergiu nas falas foi em relação a corresponsabilização do cuidado. As equipes das ESF ainda têm dificuldade de administrar as demandas de saúde mental e, por vezes, acabam não se responsabilizando pelos usuários que possuem essa demanda, por isso é necessário o psicólogo chame as ESF para responsabilização. Andrade et al. (2012) apontam que alguns profissionais que fazem parte da ESF não se comprometem em desenvolver responsabilidades compartilhadas. Isto também é representado pelo relato do psicólogo 09: "O NASF tá ali para apoiar, mas não é para fazer, não é para ser responsável, porque a responsabilidade tem que ser compactuada com a Unidade de Saúde, para que cada um faça a sua parte".

O NASF vem na proposta de apoiar as equipes de ESF e aumentar a resolutividade do cuidado, porém, identificou-se a necessidade da equipe de NASF possuir uma retaguarda profissional devido à quantidade de demandas de saúde mental. Desse modo, o profissional sente-se como o equilibrista que, busca dar apoio a equipe, porém identifica uma vasta demanda na qual não consegue resolver, repercutindo em um sentimento de frustração, de ausência de apoio para a equipe do NASF, conforme destaca a participante:

Eu acho que deveria ter tipo um psicólogo clínico geral, que seria fazer aquele papel de realizar uma triagem para dar os devidos encaminhamentos, se tivesse para onde encaminhar. Se for para um município maior, talvez tenha uns psicólogos nas pontas, mas, mesmo assim, é uma demanda gigantesca (Psicólogo 01).

De acordo com os relatos, foi possível perceber a carência de outros serviços especializados que sirvam de subsídio para esses profissionais nos atendimentos da população. Embora a Portaria n ${ }^{\circ} 3.088$, de dezembro de 2011, sobre Rede de Atenção Psicossocial não preconize a existência da retaguarda profissionais para o NASF, destaca-se a necessidade de polos que propiciem esse apoio aos profissionais inseridos no NASF (Portaria n. 3.088, 2011). Essa falta de retaguarda especializada resulta em encaminhamentos em excesso vindo de outros serviços como escolas, pois segundo os profissionais, a mesma não possui uma equipe para cessar as demandas e realizar as orientações:

Hoje a nossa educação do município não tem uma equipe multidisciplinar para fazer o acompanhamento desses alunos, [...] para daí estar direcionando quem vai ser encaminhado para o atendimento. Então, a escola hoje na verdade encaminha muito crianças e jovens para cá. A maioria deles por dificuldades de aprendizagem (Psicólogo 02). 
Outra questão levantada pelos entrevistados é a ausência de espaços de cuidado para a população, como o CAPS no município, além de três profissionais não terem sua atuação voltada especialmente para o NASF. Na existência de um serviço especializado, os profissionais quando não obtêm resolutividade em algum caso realizam encaminhamentos para locais da Atenção Secundária, como o CAPS, quando o município possui esse serviço.

Concomitante a esses aspectos, alguns profissionais criticam sobre a forma de funcionamento do NASF em seu município, como a contratação de profissionais do NASF somente para o recebimento de recursos, já que alguns profissionais acabam atuando em outro local. Outro fator levantado por um psicólogo como prejudicial foi seu trabalho estar voltado para responder por produção, onde necessita realizar encaminhamentos dos atendimentos para resultar em indicadores para o Estado, com isso a gestão do seu município acaba não utilizando esses dados para pensar em atividades estratégicas para a melhoria do processo de trabalho na rede de saúde, tal situação acaba desestimulando o profissional.

Os entrevistados frisam que a falta de profissionais prejudica a estruturação de suas ações, pois os mesmos ficam sobrecarregados diante as demandas excessivas em saúde mental, como elucidado na fala do psicólogo 02: "Somos em três psicólogas no município e estamos na falta de uma [...] tudo isso dificulta que a gente consiga focar especificamente nas áreas de atuação, isso é um fator que vem dificultando também na organização dessa prática do NASF". De acordo Azevedo e Kind (2013), os psicólogos queixam-se de não haver outro profissional da Psicologia no NASF para discutir casos, trocar ideias e tirar dúvidas, com isso, acabam sentindo-se sobrecarregados.

\section{Considerações finais}

De acordo com os objetivos da pesquisa buscou-se compreender a atuação dos psicólogos inseridos no NASF na Região de Saúde da Foz do Vale Itajaí, assim, foi possível identificar pelos resultados obtidos que a atuação do psicólogo neste serviço volta-se às práticas sanitárias, técnico-pedagógicas e clínico-assistenciais. As práticas sanitárias foram caracterizadas pelo levantamento de demandas e indicadores de saúde, bem como as formas de avaliação e monitoramento desses indicadores. Identificou-se também as ações dos profissionais que favorecem o levanta- mento das demandas, como o PMAQ e as dificuldades relacionadas à organização e monitoramento desses dados levantados.

Em relação às práticas técnico-pedagógicas, evidenciou-se que as ações realizadas direcionadas ao apoio voltam-se as atividades de educação permanente, reuniões de equipe, Projeto Terapêutico Singular, discussões de casos, bem como outras ações intersetoriais. No entanto, alguns psicólogos citam também que o apoio às equipes de ESF é inexistente, porque o NASF existe somente no Cadastro Nacional de Estabelecimento de Saúde (CNES), de modo a refletir sobre a necessidade de fiscalização tanto por parte do Conselho Municipal de Saúde, quanto das autarquias ministeriais e estaduais.

As intervenções clínico-assistenciais foram evidenciadas através dos atendimentos individuais, compartilhados e domiciliares, além de grupos de diversas modalidades. Contudo, é possível identificar muitos desafios e fragilidades na atuação do psicólogo na saúde pública, como a resistência da comunidade em aderir aos grupos, falta de conhecimento sobre a política do NASF, demanda excessiva de saúde mental, falta de profissionais e escassez de outros espaços de atenção, como o CAPS. Tais profissionais do NASF sente-se como um equilibrista, uma vez que realizam o apoio para as equipes de referência e garantem o cuidado da população, porém não possuem suporte técnico de outros locais de atenção à saúde, de modo a gerar frustrações e sobrecarga profissional. Assim, sugere-se que sejam criados polos multidisciplinares de saúde para dar retaguarda aos profissionais do NASF.

Desta forma, frente aos resultados, sugere-se que sejam realizadas reuniões com os gestores, a fim de refletir sobre política do NASF, tendo em vista as solicitações de intervenções da gestão para os profissionais que atuam nesse núcleo, em alguns casos ainda são voltadas somente para o atendimento clínico, não sendo condizentes com o que é proposto pelo Ministério da Saúde. Tais realidades trazem à tona a necessidade de repensar a dimensão assistencial do apoio matricial, pois alguns psicólogos mesmo pertencendo a equipe do NASF estão alocados em serviços de atenção especializada, a fim de dar conta da alta demanda de saúde mental dentro do contexto clínico-assistencial.

Além disso, seria necessário que os psicólogos do NASF estabelecessem protocolos de compartilha- 
mento de casos para criar critérios para a realização do atendimento individual, com o intuito de atender somente os casos necessários, além de criar linhas de cuidado para possibilitar que o trabalho em rede seja efetivado. Destaca-se também a necessidade da participação da Psicologia nas reuniões dos Conselhos Municipais de Saúde, a fim de lutar para implantação de serviços que possibilitem a concretização do NASF, como por exemplo, a criação de polos multidisciplinares para dar a retaguarda para o NASF, colaborando para a efetivação do apoio matricial.

Por fim, sugere-se para futuras pesquisas investigar as ações de NASF a partir da perspectiva dos gestores de saúde e de usuários, para de refletir sobre esses atores que também fazem parte do processo de implantação e implementação do NASF.

\section{Referências}

Andrade, L. M. B., Quandt, F. L., Campos, D. A., Delziovo, C. R., Coelho, E. B. S., \& Moretti-Pires, R. O. (2012). Análise da implantação dos núcleos de apoio à saúde da família no interior de Santa Catarina. Saúde \& Transformação Social, 3(1), 18-31.

Azevedo, N. S., \& Kind, L. (2013). Psicologia nos núcleos de apoio à saúde da família em Belo Horizonte. Psicologia: Ciência e Profissão, 33(3), 520-535. https://doi.org/10.1590/S1414-98932013000300002

Brasil. Ministério da Saúde. (2014). Cadernos de atenção básica: Núcleo de apoio à saúde da família. Brasília, DF: Ministério da Saúde. Recuperado de: http://dab.saude.gov.br/portaldab/biblioteca.php?conteudo=publicacoes/cab39.

Brasil. Ministério da saúde. (2012). Programa nacional de melhoria do acesso e da qualidade da atenção básica (PMAQ): Manual instrutivo. Brasília, DF: Ministério da Saúde. Recuperado de: http://dab.saude.gov.br/portaldab/biblioteca.php?conteudo=publicacoes/pmaq.

Campos, G. W. S., \& Domitti, A. C. (2007). Apoio matricial e equipe de referência: Uma metodologia para gestão do trabalho interdisciplinar em saúde. Cadernos de Saúde Pública, 23(2) 399-407. https://doi.org/10.1590/S0102$311 X 2007000200016$

Cassiani, S. H. B., \& Almeida, A. M. (1999). Teoria fundamentada nos dados: A coleta e análise de dados qualitativos. Cogitare Enfermagem, 4(2), 13-21. https://doi.org/10.5380/ce.v4i2.44840

Cezar, P. K., Rodrigues, P. M., \& Arpini, D. M. (2015). A psicologia na estratégia de saúde da família: Vivências da residência multiprofissional. Psicologia: Ciência e Profissão, 35(1), 221-224. https://doi.org/10.1590/19823703000012014

Cord, D., Gesser, M., Nunes, A. S. B., \& Storti, M. M. T. (2015). As significações de profissionais que atuam no programa saúde na escola (PSE) acerca das dificuldades de aprendizagem: Patologização e medicalização do fracasso escolar. Psicologia: Ciência e Profissão, 35(1), 40-53. https://doi.org/10.1590/1982-3703000952013

Friese, S. (2013). ATLAS.ti 7: User guide and reference. Berlin: Atlas.ti. Recuperado de: http://atlasti.com/wp-content/uploads/2014/05/atlasti_v7_manual_201312.pdf?q=/uploads/media/atlasti_v7_manual_201312.pdf

Furtado, M. E. M. F., \& Carvalho, L. B. (2015). O psicólogo no NASF: Potencialidades e desafios de um profissional de referência. Revista Psicologia e Saúde, 7(1), 9-17.

Gonçalves, R. M. A., Lancman, S., Sznelwar, L. I., Cordone, N. G., \& Barros, J. O. (2015). Estudo do trabalho em núcleos de apoio à saúde da família (NASF), São Paulo, Brasil. Revista Brasileira da Saúde Ocupacional, 40(131), 59-74. https:// doi.org/10.1590/0303-7657000078013

Gryschek, G., \& Pinto, A. A. M. (2015). Saúde mental: como as equipes de saúde da família podem integrar esse cuidado na atenção básica? Ciência \& Saúde Coletiva, 20(10), 3255-3262. https://doi.org/10.1590/1413812320152010.13572014

Hori, A. A., \& Nascimento, A. F. (2014). O projeto terapêutico singular e as práticas de saúde mental nos núcleos de apoio à saúde da família (NASF) em Guarulhos (SP), Brasil. Ciência \& Saúde Coletiva, 19(8), 3561-3571. https://doi.org/10.1590/1413-81232014198.11412013

Lancman, S., Gonçalves, R. M. A., Cordone, N. G., \& Barros, J. O. (2013). O estudo do trabalho e do trabalhar no núcleo de apoio à saúde da família. Revista de Saúde Pública, 47(5), 968-975. https://doi.org/10.1590/S00348910.2013047004770 
Lucena, M., Mourão, H., \& Nepomuceno, L. (2009). A prática da psicologia no NASF. In: Conselho Federal de Psicologia, A prática da psicologia e o núcleo de apoio à saúde da família (pp. 31-49). Brasília, DF: Conselho Federal de Psicologia. Recuperado de: http://site.cfp.org.br/wp-content/uploads/2009/12/Seminxrio_O_Nxcleo_de_ Apoio-beta.pdf

Malta, D. C., Morais, O. L. M., Neto, Silva, M. M. A., Rocha, D., Castro, A. M., Reis, A. A. C. et al. (2016). Política nacional de promoção da saúde (PNPS): Capítulos de uma caminhada ainda em construção. Ciência \& Saúde Coletiva, 21(6), 1683-1694. https://doi.org/10.1590/1413-81232015216.07572016

Perrella, A. C. (2015). A experiência da psicologia no NASF: Capturas, embates e invenções. Revista Interinstitucional de Psicologia, 8(2), 443-452.

Pope, C., \& Mays, N. (2009). Métodos qualitativos na pesquisa em saúde. In C. Pope, \& N. Mays (Orgs.), Pesquisa qualitativa na atenção à saúde (pp. 207-223). Porto Alegre, RS: Artmed.

Portaria GM n. 154, de 24 de janeiro de 2008. Cria os núcleos de apoio à saúde da família NASF. Recuperado de: http://dab.saude.gov.br/docs/legislacao/portaria154_24_01_08.pdf

Portaria n. 3.088, de 23 de dezembro de 2011. Institui a rede de atenção psicossocial para pessoas com sofrimento ou transtorno mental e com necessidades decorrentes do uso de crack, álcool e outras drogas, no âmbito do Sistema Único de Saúde (SUS). Recuperado de: http://bvsms.saude.gov.br/bvs/saudelegis/gm/2011/ prt3088_23_12_2011_rep.html

Portaria n. 3.124, de dezembro de 2012. Redefine os parâmetros de vinculação dos núcleos de apoio à saúde da família (NASF) modalidades 1 e 2 às equipes saúde da família e/ou equipes de atenção básica para populações específicas, cria a Modalidade NASF 3, e dá outras providências. Recuperado de: http://bvsms.saude.gov.br/bvs/ saudelegis/gm/2012/prt3124_28_12_2012.html

Reis, M. L., Medeiros, M., Pacheco, L. R., \& Caixeta, C. C. (2016). Avaliação do trabalho multiprofissional do núcleo de apoio à saúde da família. Texto \& Contexto Enfermagem, 25(1), 1-9. https://doi.org/10.1590/0104-070720160002810014

Santos, A. L., \& Rigotto, R. M. (2010). Território e territorialização: Incorporando as relações produção, trabalho, ambiente e saúde na atenção básica à saúde. Trabalho Educação e Saúde, 8(3), 387-406. https://doi.org/10.1590/ S1981-77462010000300003

Tavares, S. M. G. (2008). A implementação dos núcleos de apoio à saúde da família (NASF) e a inserção da saúde mental no território NASF/ESF. BIS: Boletim Instituto de Saúde, (45), 10-12.

\section{Roberta Borghetti Alves}

Mestre em Psicologia pela Universidade Federal de Santa Catarina. Docente da Universidade do Vale do Itajaí (Univali), Itajaí - SC. Brasil.

E-mail: roberta_alves@univali.br. http://orcid.org/0000-0002-1866-699X

\section{Natália de Oliveira Bruning}

Graduada em Psicologia na Universidade do Vale do Itajaí (Univali), Itajaí - SC. Brasil.

E-mail: nataliabruning@hotmail.com.

http://orcid.org/0000-0001-8751-7907

\section{Ketillyn Cristina Kohler}

Graduada em Psicologia na Universidade do Vale do Itajaí (Univali), Itajaí - SC. Brasil.

E-mail: ketillyn_@hotmail.com.

http://orcid.org/0000-0003-3754-7950

Endereço para envio de correspondência:

Universidade do Vale do Itajaí. Curso de Psicologia. Bloco F2, Sala 401.

Rua Uruguai, Centro. CEP: 888302-202. Itajaí - SC. Brasil. 
Recebido 16/10/2017

Reformulado 26/06/2018

Aceito 23/08/2018

Received 10/16/2017

Reformulated $06 / 26 / 2018$

Approved 08/23/2018

Recibido $16 / 10 / 2017$

Reformulado 26/06/2018

Aceptado 23/08/2018

Como citar: Alves, R. B.; Bruning, N. O.; Kohler, K. C. (2019). “O equilibrista”: atuação do psicólogo no NASF no Vale do Itajaí. Psicologia Ciência e Profissão, 39, 1-13. https://doi.org/10.1590/ 1982-3703003186600

How to cite: Alves, R. B.; Bruning, N. O.; Kohler, K. C. (2019). “The equilibrist”: performance of the psychologist in the NASF in the Vale of Itajaí. Psicologia Ciência e Profissão, 39, 1-13. https://doi.org/10.1590/1982-3703003186600

Cómo citar: Alves, R. B.; Bruning, N. O.; Kohler, K. C. (2019). "El equilibrista": actuación del psicólogo en el NASF en el Valle do Itajaí. Psicologia Ciência e Profissão, 39, 1-13. https://doi.org/10.1590/1982-3703003186600 